\title{
Losing the trace to find dynamical Newton or Planck constants
}

\author{
Pavel Jiroušek, ${ }^{1,2, *}$ Keigo Shimada, ${ }^{3, \dagger}$ Alexander Vikman, ${ }^{1,}{ }^{\ddagger}$ and Masahide Yamaguchi ${ }^{3,} \S$ \\ ${ }^{1}$ CEICO-Central European Institute for Cosmology and Fundamental Physics, \\ FZU-Institute of Physics of the Czech Academy of Sciences, \\ Na Slovance 1999/2, 18221 Prague 8, Czech Republic \\ ${ }^{2}$ Institute of Theoretical Physics, Faculty of Mathematics and Physics, Charles University, \\ $V$ Holešovičkách 2, 18000 Prague 8, Czech Republic \\ ${ }^{3}$ Department of Physics, Tokyo Institute of Technology, Tokyo 152-8551, Japan
}

(Dated: April 16, 2021)

\begin{abstract}
We show that promoting the trace part of the Einstein equations to a trivial identity results in the Newton constant being an integration constant. Thus, in this formulation the Newton constant is a global dynamical degree of freedom which is also a subject to quantization and quantum fluctuations. This is similar to what happens to the cosmological constant in the unimodular gravity where the trace part of the Einstein equations is lost in a different way. We introduce a constrained variational formulation of these modified Einstein equations. Then, drawing on analogies with the HenneauxTeitelboim action for unimodular gravity, we construct different general-covariant actions resulting in these dynamics. The inverse of dynamical Newton constant is canonically conjugated to the Ricci scalar integrated over spacetime. Surprisingly, instead of the dynamical Newton constant one can formulate an equivalent theory with a dynamical Planck constant. Finally, we show that an axion-like field can play a role of the gravitational Newton constant or even of the quantum Planck constant.
\end{abstract}

\section{INTRODUCTION AND OUR MAIN IDEA}

The origin of the cosmological constant and its relation to the vacuum fluctuations of quantum fields persists to be one of the main puzzles of contemporary physics, see e.g. [1-4]. However, since Einstein's seminal paper [5] it is well known that trace-free equations

$$
G_{\mu \nu}-\frac{1}{4} g_{\mu \nu} G=8 \pi G_{N}\left(T_{\mu \nu}-\frac{1}{4} g_{\mu \nu} T\right),
$$

result in a cosmological constant (CC), $\Lambda$, as a constant of integration, or a global degree of freedom, see e.g. [2, 611]. For all other purposes, the trace-free equations above are entirely classically equivalent to the usual General Relativity (GR) equations with $\Lambda$

$$
G_{\mu \nu}=8 \pi G_{N} T_{\mu \nu}+\Lambda g_{\mu \nu} .
$$

Crucially, the trace-free Einstein equations (1) are invariant under vacuum shifts of the total energymomentum tensor (EMT)

$$
T_{\mu \nu} \rightarrow T_{\mu \nu}+c g_{\mu \nu}
$$

with $c=c\left(x^{\mu}\right)$, and in particular with $c=$ const, which is not the case for the usual Einstein equations (2), with or without $\Lambda$.

Nothing illustrates better the currently unexplainable small value of $\Lambda$, as a comparison with $G_{N}$ - the only

\footnotetext{
*Electronic address: jirousek@fzu.cz

$\dagger$ Electronic address: shimada.k.ah@m.titech.ac.jp

¥Electronic address: vikman@fzu.cz

$\S$ Electronic address: gucci@phys.titech.ac.jp
}

other dimensionful ${ }^{1}$ constant in (2):

$$
G_{N} \Lambda \sim 10^{-122}
$$

The usual question to ask regarding these 122 orders of magnitude is: why is $\Lambda$ so vanishingly small, but still not entirely zero? Perhaps one should invert the question and ask instead: why is $G_{N}$ that tiny? Both these questions require an ability to select values on these constants from some ensemble. Thus, it makes sense to look for simple theories where $G_{N}$ would appear as an integration constant or a global degree of freedom. This is a "landscape" of poor people.

With this motivation in mind, in this paper we propose a novel way to lose the trace part of the Einstein equations. Indeed, instead of (1) one can write normalized or trace-trivial equations

$$
\frac{G_{\mu \nu}}{G}=\frac{T_{\mu \nu}}{T} .
$$

These equations are scale-free and invariant with respect to rescaling ${ }^{2}$

$$
T_{\mu \nu} \rightarrow c T_{\mu \nu}
$$

by a constant, or even an arbitrary function $c\left(x^{\mu}\right)$. As we show in this paper, equations (5) are again equivalent

\footnotetext{
${ }^{1}$ We use $(+,-,-,-)$ signature convention and units where $c=1$, while in most parts of the text $\hbar=1$ and we restore $\hbar$ only to stress the quantum nature of a formula.

2 It is worth noting that transformations (3) and (6) could be performed on the Einstein tensor instead of the EMT, see e.g. [12]. However, it is not clear how to realize these transformations as transformations of the metric. Moreover, the equations are completely blind to independent transformations of both tensors.
} 
to the usual GR, however now $G_{N}$ becomes a constant of integration.

Following Einstein [5] on the cosmological constant, one can either conclude that "... the new formulation has this great advantage, that the quantity appears in the fundamental equations as a constant of integration, and no longer as a universal constant peculiar to the fundamental law" or rather that "Since the world exists as a single specimen, it is essentially the same whether a constant is given the form of one belonging to the natural laws or the form of an 'integration constant'", as in $[13]^{3}$. Crucially, quantum cosmology and chaotic self-reproducing inflation $[15,16]$ allow for a multiverse so that the world would be not a "single specimen", as thought by Einstein. Thus, one can speculate that the value of the Newton constant may be a remnant from the early universe quantum gravity era, similarly to what could happen to the cosmological constant, as discussed in e.g. [17, 18].

\section{RECAP OF UNIMODULAR GRAVITY}

Our proposal shares some structural similarities with the unimodular gravity. Hence, it is worthwhile to recall its basic properties here. As described in e.g. [2, 6-11], one can apply covariant derivative $\nabla_{\mu}$ to both sides of (1) and by virtue of the Bianchi identity, $\nabla_{\mu} G^{\mu \nu}=0$, along with the assumed conservation of EMT, $\nabla_{\mu} T^{\mu \nu}=0$, one obtains $^{4}$

$$
\partial_{\mu}(G-\varkappa T)=0,
$$

where we introduced notation

$$
\varkappa=8 \pi G_{N},
$$

to make later expressions shorter. In this way

$$
G-\varkappa T=4 \Lambda
$$

where $\Lambda$ is a constant of integration. Substitution of this differential consequence back into the trace-free Einstein equations (1) yields normal Einstein's General Relativity (GR) with the cosmological constant $\Lambda$. Thus, loosing the trace-part of the standard Einstein equations in this way results in promoting (or demoting) the cosmological constant (CC) from the fundamental constants of nature, parameters or coupling constants to a global dynamical degree of freedom. This modification of GR is known under the name "unimodular gravity".

One can obtain the traceless Einstein equations (1), provided one varies the Einstein-Hilbert action under

\footnotetext{
${ }^{3}$ For history of the cosmological constant see e.g. [14]

${ }^{4}$ In some formulations of gravity, the Bianchi identity may not hold which results in non-vanishing right hand side of this differential consequence. The implications of this have been recently explored in [19-21].
}

constrained or restricted variation of the contravariant metric, $\bar{\delta} g^{\mu \nu}$, satisfying

$$
g_{\mu \nu} \bar{\delta} g^{\mu \nu}=0
$$

This variational constraint implies that only transverse variations $\bar{\delta} g^{\mu \nu}$ are allowed. This general-covariant restriction (10) of the variations $\delta g^{\mu \nu}$ can be obtained from an apparently non-covariant "unimodular" constraint

$$
\sqrt{-g} \equiv \sqrt{-\operatorname{det} g_{\mu \nu}}=\sigma(x)
$$

where $\sigma\left(x^{\mu}\right)$ is an unspecified function which is often taken to be just unity:

$$
\sqrt{-g}=1
$$

However, the later choice manifestly breaks general covariance and would select preferred coordinate systems. Moreover, this selection clearly excludes writing most important solutions of GR like the Schwarzschild and Friedmann metrics in natural coordinates which have simple physical interpretation and which can definitely be formed by test bodies and clocks. Clearly, for every metric, $g_{\mu \nu}$, there is enough gauge freedom to find a coordinate system to satisfy the "unimodular" constraint (12), but it is especially easy if one does not specify the function $\sigma(x)$ at all. The irrelevant choice of this function corresponds to a gauge freedom responsible for the diffeomorphism invariance. In the manifestly general-covariant formulation of the unimodular gravity by Henneaux-Teitelboim $[22]^{5}$ the meaning of the "unimodular" constraint is reversed: it is used not to find $\sqrt{-g}$ for a given $\sigma(x)$, but to find instead a dynamical variable composing $\sigma$ for a given $\sqrt{-g}$. Most importantly, restriction on variations (10) is covariant and so are the resulting traceless equations of motion (1). Thus, it is rather counterproductive to take $\sqrt{-g}=1$ and break their general covariance by this unnecessary assumption, even though this choice makes the action polynomial [26] and may simplify some calculations.

To quickly see that (10) results in (1) one writes first variation form of the Einstein equations for general variations of the metric which is also satisfied by the restricted variations $\bar{\delta} g^{\mu \nu}$

$$
\left(G_{\mu \nu}-\varkappa T_{\mu \nu}\right) \bar{\delta} g^{\mu \nu}=0
$$

However, due to the transversality constraint (10) only tensor components not "proportional" to $g_{\mu \nu}$ had to be equal to zero now. This results in appearance of the Lagrange multiplier $\lambda\left(x^{\mu}\right)$ responsible for the "reaction force" of the constraint

$$
G_{\mu \nu}-\varkappa T_{\mu \nu}=\lambda(x) g_{\mu \nu}
$$

\footnotetext{
5 For other diff invariant formulations of the unimodular gravity, see e.g. [23-25]
} 
This is an analog of the Lagrange's equations of the first kind in analytical mechanics. One can exclude unspecified Lagrange multiplier by taking the trace of (14), so that $\lambda(x)=(G-\varkappa T) / 4$, and then substituting this expression back to (14) one obtains trace-free Einstein equations (1). Of course, we could apply covariant derivative and use Bianchi identity along with the covariant conservation of EMT directly in (14) to find that $\lambda(x)=$ const .

It is well known how to write an action for the tracefree Einstein equations (1). The arguably simplest covariant action was introduced by Henneaux and Teitelboim in $[22]$

$$
S[g, W, \Lambda]=\frac{1}{\varkappa} \int d^{4} x \sqrt{-g}\left[-\frac{R}{2}+\Lambda\left(\nabla_{\mu} W^{\mu}-1\right)\right] .
$$

The second term in the brackets has a form of the Hamiltonian (or first order) action. Furthermore, variation with respect to the spatial components $W^{i}$ yields $\partial_{i} \Lambda=0$, along every Cauchy hypersurface $\Sigma$. Hence, for every imposed foliation, $\Lambda$ is space independent - is a global quantity. Using this constraint and following Faddeev-Jackiw [27, 28], one concludes "without tears" that $\Lambda / \varkappa$ is a canonical momentum conjugated to the cosmic time

$$
\mathscr{T}(t)=\int_{\Sigma} d^{3} \mathbf{x} \sqrt{-g} W^{t}(t, \mathbf{x}) .
$$

This variable measures four-volume of space-time between Cauchy hypersurfaces $\Sigma_{2}$ and $\Sigma_{1}$ as

$$
\mathscr{T}\left(t_{2}\right)-\mathscr{T}\left(t_{1}\right)=\int_{\mathscr{V}} d^{4} x \sqrt{-g} .
$$

One can think about $\mathscr{T}$ as a charge which is not conserved, but rather continuously produced by a unit source due to the constraint

$$
\nabla_{\mu} W^{\mu}=1 .
$$

As $\Lambda / \varkappa$ and $\mathscr{T}$ are canonically conjugated, one can apply Heisenberg uncertainty relation

$$
\delta \Lambda \times \delta \int_{\mathscr{V}} d^{4} x \sqrt{-g} \geq 4 \pi \ell_{P l}^{2},
$$

where $\ell_{P l}=\sqrt{\hbar G_{N}}$ is the Planck length. The presence of these quantum fluctuations is the main difference of "unimodular" gravity from the usual GR. These fluctuations may be relevant close to singularities. Except of this phenomenon, perturbative "unimodular" gravity is equivalent to GR also in quantum realm, for recent discussions see e.g. [29-42].

The constraint part of the action (15) can be written as

$$
\int d^{4} x \Lambda\left[\partial_{\mu}\left(\sqrt{-g} W^{\mu}\right)-\sqrt{-g}\right]
$$

so that $\partial_{\mu}\left(\sqrt{-g} W^{\mu}\right)$ plays the role of the unspecified function $\sigma(x)$ in (11) or the measure ${ }^{6}$ from [6]. Though, now the "unimodular" constraint (11) is used to find $W^{\mu}$ without imposing any restrictions on the metric.

On the other hand, action (15) possesses a gauge redundancy

$$
W^{\mu} \rightarrow W^{\mu}+\epsilon^{\mu} \text {, where } \nabla_{\mu} \epsilon^{\mu}=0 .
$$

For every $W^{\mu}$ and every choice of coordinates $\left(t^{\prime}, x^{i}\right)$ one can find such $\epsilon^{\mu}$ that the spatial components vanish $W^{\mu}=\left(W^{t^{\prime}}, 0\right)$. Further, one can introduce new coordinates $\left(t, x^{i}\right)$ with $t\left(t^{\prime}, x^{i}\right)=\sqrt{-g^{\prime}} W^{t^{\prime}}$, so that

$$
W^{t}=\frac{\partial t}{\partial t^{\prime}} W^{t^{\prime}}=\frac{\partial t}{\partial t^{\prime}} \frac{t}{\sqrt{-g^{\prime}}},
$$

which through the transformation of measure $\sqrt{-g} \partial t / \partial t^{\prime}=\sqrt{-g^{\prime}}$ implies that

$$
W^{\mu}=\delta_{t}^{\mu} \frac{t}{\sqrt{-g}} .
$$

If we fix this gauge and these coordinates before performing the variation (what is usually not a correct way to proceed) we obtain the often used "unimodular" constraint $^{7}$

$$
\int d^{4} x \Lambda[1-\sqrt{-g}]
$$

which is a usual starting point for a non-covariant formulation of the unimodular gravity [26, 52-54]. Sometimes it is claimed that this constraint (24) can be satisfied just by a proper choice of the coordinates. However, it is worth emphasizing that on the way to $(24)$ we have already used the diffeomorphism invariance once to enforce the unity of the density $\partial_{\mu}\left(\sqrt{-g} W^{\mu}\right)$. If we could use the diffeomorphism again to enforce the unity of $\sqrt{-g}$, this would imply that just by a diffeomorphism one could enforce the unity of the scalar $\nabla_{\mu} W^{\mu}$ which is clearly not possible. Thus, constraint (24) is not just a mere gauge choice, but does change the dynamics by introducing a global degree of freedom.

As showed in [25], the vector field $W^{\mu}$ can be exchanged with a more convenient Chern-Simons current of a (abelian or non-abelian) gauge field $A_{\mu}$ so that

$$
S[g, A, \Lambda]=\frac{1}{\varkappa} \int d^{4} x \sqrt{-g}\left[-\frac{R}{2}+\Lambda\left(F_{\alpha \beta} F^{\star \alpha \beta}-1\right)\right],
$$

\footnotetext{
${ }^{6}$ Interestingly, this can be considered as a particular example of theories with two different measures, see e.g. [43-45].

7 In terms of the ADM variables $[46,47]$ the "unimodular" constraint relates the lapse $N$ to the determinant of the spatial metric $\gamma$ as $N=\gamma^{-1 / 2}$. This relation can be further extended [48] to a general function $N=f(\gamma)$, with rather peculiar consequences, e.g. [49-51].
} 
where $F_{\mu \nu}=D_{\mu} A_{\nu}-D_{\nu} A_{\mu}$ is the gauge field strength or curvature of the covariant derivative $D_{\mu}=\nabla_{\mu}+i q A_{\mu}$, while the Hodge dual is defined as

$$
F^{\star \alpha \beta}=\frac{1}{2} \cdot \frac{\epsilon^{\alpha \beta \mu \nu}}{\sqrt{-g}} \cdot F_{\mu \nu} \equiv \frac{1}{2} \cdot E^{\alpha \beta \mu \nu} \cdot F_{\mu \nu} .
$$

Moreover, one could avoid the Lagrange multiplier and formulate unimodular gravity as a higher-derivative and Weyl-invariant theory of a vector field [24] or of a gauge field [25], see also [55]. One can also describe this dynamics by a covariant action containing a three-form as in e.g. [56, 57], but we will not expand on that.

Finally, we would like to mention that it is the absence of the usual kinetic term $F_{\alpha \beta} F^{\alpha \beta}$ which forces $\Lambda$ to be constant. Hence, it is easy to further extend this action to resemble the one of the usual axion, c.f. [58] and see [25]:

$$
\begin{aligned}
& S[g, A, \theta]=\int d^{4} x \sqrt{-g}\left[-\frac{R}{2 \varkappa}+\right. \\
& \left.+\frac{1}{2}(\partial \theta)^{2}+\frac{\theta}{f_{\Lambda}} F_{\alpha \beta} F^{\star \alpha \beta}-V_{\lambda}(\theta)\right],
\end{aligned}
$$

where now $\theta$ is a canonically normalized pseudoscalar and $f_{\Lambda}$ is some mass scale emulating axion decay constant and $V_{\lambda}(\theta)$ is a "potential". The presence of the standard kinetic term for $\theta$ does not change anything, as on-shell $\theta$ is always a constant $\theta_{\star}$ corresponding to the vacuum energy density $V_{\lambda}\left(\theta_{\star}\right)$. In particular, this action is parity symmetric. This gives a hope to find unimodular gravity as a particular dynamical regime (maybe strongly coupled) of some more usual high energy system.

\section{SCALE-FREE EQUATIONS}

In this paper we propose a novel way to lose the trace part of the Einstein equations. Indeed, instead of (1) one can write normalized or scale-free and still trace-trivial equations

$$
\frac{G_{\mu \nu}}{G}=\frac{T_{\mu \nu}}{T}
$$

so that, the trace part of these normalized Einstein equations becomes a useless identity $1=1$ instead of $0=0$ in the previous "unimodular" case. This unitrace formulation does not require any dimensional parameter, as both sides are dimensionless. If again $T_{\mu \nu}$ is covariantly conserved, one can follow the same procedure as for the trace-free Einstein equations (1). We apply covariant derivative to both sides of (28) and use Bianchi identity to obtain

$$
\frac{G_{\mu \nu}}{G^{2}} \nabla^{\mu} G=\frac{T_{\mu \nu}}{T^{2}} \nabla^{\mu} T .
$$

Substituting here the normalized Einstein equations (28) one obtains instead of (7)

$$
\partial_{\mu} \log \frac{G}{T}=0,
$$

so that

$$
G=\beta T,
$$

where $\beta$ is constant of integration. Now we can substitute this differential consequence (31) back into the original normalized Einstein equations (28) to obtain the standard Einstein equations

$$
G_{\mu \nu}=\beta T_{\mu \nu}
$$

Thus, this novel trace-trivial formulation (28) allows to have the Newton constant

$$
G_{N}=\frac{\beta}{8 \pi},
$$

as a constant of integration or global dynamical degree of freedom. Similarly to $\Lambda$ in the "unimodular" case, this constant of integration $\beta$ is allowed to be both positive and negative.

Here a cautionary remark is necessary: not only we assumed that the traces $T$ and $G$ are non-vanishing, but also we required a stronger condition that $G_{\mu \nu}$ (or $T_{\mu \nu}$ ) has an inverse for interesting solutions. Of course gravity waves in empty space would violate this assumption. However, conformal anomaly can motivate the nonvanishing trace of the $\mathrm{EMT}^{8}$, while an existence of an arbitrary small cosmological constant (included into this EMT) would imply desired invertibility for all solutions, except of measure zero when this CC is exactly compensated by some matter.

Interestingly, one can obtain normalized, scale-free and trace-trivial equations (28) from a restricted variation of the Einstein-Hilbert action. However, now instead of the transversality condition (10) one should take either "Einstein transversality" condition:

$$
G_{\mu \nu} \bar{\delta} g^{\mu \nu}=0,
$$

or the "energy transversality" condition:

$$
T_{\mu \nu} \bar{\delta} g^{\mu \nu}=0 .
$$

These conditions are mutually complementary and interchangeable. Indeed, assuming one of them results through the general unrestricted variational relation (13) in the other one. Either of them would be deadly for unrestricted variations, but here we should merely require equality of only those parts of $T_{\mu \nu}$ which are not proportional to $G_{\mu \nu}$. Namely, imposing the "Einstein transversality" condition (13) yields the Lagrange equations of the first kind

$$
\varkappa T_{\mu \nu}=\lambda(x) G_{\mu \nu}
$$

\footnotetext{
8 In particular, the trace of total EMT and the corresponding Ricci scalar are not entirely vanishing during the early universe radiation domination époque, see e.g. [59].
} 
where $\lambda(x)$ is a Lagrange multiplier. Now we can again multiply both sides of this equation with $g^{\mu \nu}$ and exclude the Lagrange multiplier to obtain (28). Clearly, the "energy transversality" case is treated completely analogously. Similarly to the unimodular gravity we could apply covariant derivative and use Bianchi identity along with the assumed covariant conservation of EMT directly in $(36)$ to find that $\lambda(x)=$ const.

\section{MANY FACES OF LAGRANGE}

The proportionality between $G_{\mu \nu}$ and $T_{\mu \nu}$ in our scalefree formulation, or proportionality between the metric $g_{\mu \nu}$ and $\left(G_{\mu \nu}-\varkappa T_{\mu \nu}\right)$ in the case of the unimodular gravity, can be expressed without any use of the Lagrange multiplier. One can follow an analogy with usual vectors. There, any two vectors are proportional, provided their vector (wedge) product vanishes and vice versa. Thus, for our novel scale-free gravity one can write analogous expression

$$
G_{\mu \nu} T_{\alpha \beta}=G_{\alpha \beta} T_{\mu \nu}
$$

instead of the Lagrange's equations of the first kind for the scale-free formulation (36), while for the unimodular case one would write

$$
\left(G_{\mu \nu}-\varkappa T_{\mu \nu}\right) g_{\alpha \beta}=\left(G_{\alpha \beta}-\varkappa T_{\alpha \beta}\right) g_{\mu \nu},
$$

instead of (14).

Further, it is noteworthy that one can exclude the Lagrange multipliers from the Lagrange equations of the first kind (14), (36) in many different ways. The multiplication of (14), (36) with respect to the contravariant metric has a clear advantage that this inverse metric always exists and that it's trace is always equal to the number of spacetime dimensions. However, one could use other equivalent ways to eliminate the Lagrange multiplier and write equations in terms of known fields in different forms.

\section{Unimodular gravity}

For example, in unimodular gravity one could multiply (14) with $G^{\mu \nu}$ and, under the assumption that $G \neq 0$, obtain

$$
\lambda=\frac{G^{\mu \nu}}{G}\left(G_{\mu \nu}-\varkappa T_{\mu \nu}\right)
$$

so that

$$
G_{\mu \nu}-\varkappa T_{\mu \nu}=\frac{G^{\alpha \beta}}{G}\left(G_{\alpha \beta}-\varkappa T_{\alpha \beta}\right) g_{\mu \nu} .
$$

Or repeating the same procedure with $T_{\mu \nu}$

$$
G_{\mu \nu}-\varkappa T_{\mu \nu}=\frac{T^{\alpha \beta}}{T}\left(G_{\alpha \beta}-\varkappa T_{\alpha \beta}\right) g_{\mu \nu} .
$$

Clearly, one could take any second rank tensor $M^{\alpha \beta}$ constructed from the matter fields and the metric and then use it instead of $T^{\alpha \beta}$ or $G^{\alpha \beta}$, provided the trace $M \neq 0$.

On the other hand, instead of taking the trace one can take the determinant of (14) to obtain

$$
\lambda^{4}=\frac{\operatorname{det}\left(G_{\mu \nu}-\varkappa T_{\mu \nu}\right)}{\operatorname{det} g_{\alpha \beta}} .
$$

Consequently for positive $\lambda$ instead of trace-free Einstein equations (1) of the unimodular gravity one acquires equations on unimodular parts of the corresponding tensors

$$
\frac{G_{\mu \nu}-\varkappa T_{\mu \nu}}{\left(-\operatorname{det}\left(G_{\alpha \beta}-\varkappa T_{\alpha \beta}\right)\right)^{1 / 4}}=\frac{g_{\mu \nu}}{\left(-\operatorname{det} g_{\alpha \beta}\right)^{1 / 4}} .
$$

Of course, taking the trace of these equations one excludes determinants and arrives back to trace-free Einstein equations (1).

\section{Scale-free gravity}

Similarly one can write the scale-free Einstein equations (28) in many different ways. For example, analogously to the unimodular case one can use determinant to solve for the Lagrange multiplier in the unitrace case and write

$$
\frac{G_{\mu \nu}}{\left(\operatorname{det} G_{\alpha \beta}\right)^{1 / 4}}=\frac{T_{\mu \nu}}{\left(\operatorname{det} T_{\alpha \beta}\right)^{1 / 4}},
$$

so that again there is no scale - no dimensionful parameter. It looks like it is a very different equation. However, taking the trace one acquires that

$$
\frac{\left(\operatorname{det} T_{\mu \nu}\right)^{1 / 4}}{\left(\operatorname{det} G_{\mu \nu}\right)^{1 / 4}}=\frac{T}{G}
$$

so that one can get rid of the ratio of the determinants and obtains in this way the same (28). This different parametrization can be as general as

$$
\frac{G_{\mu \nu}}{f\left(G_{\alpha \beta}\right)}=\frac{T_{\mu \nu}}{f\left(T_{\alpha \beta}\right)},
$$

where $f$ is general homogeneous function of the first degree of the tensor argument. In that case, there is no scale in this equation. This function can also depend on the metric. Instead of the built-in identity $1=1$ with respect to taking the trace ${ }^{9}$ of (28), here the corresponding identity is $f\left(G_{\mu \nu} / f\left(G_{\alpha \beta}\right)\right)=1$. However, taking the

\footnotetext{
${ }^{9}$ Note that the operation of taking trace can be considered as a particular example of such function on tensors, $f\left(\mathcal{O}_{\alpha \beta}\right)=$ $g^{\alpha \beta} \mathcal{O}_{\alpha \beta}$, which is homogeneous of the first degree.
} 
trace one gets back (28) again. For example,

$$
\frac{G_{\mu \nu}}{(-g)^{1 / 4} G+\left(\operatorname{det} G_{\alpha \beta}\right)^{1 / 4}}=\frac{T_{\mu \nu}}{(-g)^{1 / 4} T+\left(\operatorname{det} T_{\alpha \beta}\right)^{1 / 4}},
$$

still reproduces exactly the same dynamics as (28). Similarly to the unimodular case, one can also use $G^{\mu \nu}$ to express the Lagrange multiplier in (36) as

$$
\lambda(x)=\varkappa \frac{T_{\mu \nu} G^{\mu \nu}}{G^{\alpha \beta} G_{\alpha \beta}} .
$$

In this way, instead of unitrace equations (28) one arrives to scale-free equations

$$
T_{\mu \nu}=\frac{T_{\sigma \lambda} G^{\sigma \lambda}}{G^{\alpha \beta} G_{\alpha \beta}} G_{\mu \nu},
$$

with built-in identity with respect to multiplication with $G^{\mu \nu}$. On the other hand, multiplying (36) with $T^{\mu \nu}$ one obtains

$$
\frac{T_{\sigma \lambda} G^{\sigma \lambda}}{T^{\alpha \beta} T_{\alpha \beta}} T_{\mu \nu}=G_{\mu \nu} .
$$

Again, instead of $T_{\mu \nu}$ or $G_{\mu \nu}$ one could employ any second rank tensor $M^{\alpha \beta}$ constructed from the matter fields and metric, provided the trace $M^{\alpha \beta} G_{\alpha \beta} \neq 0$, so that

$$
\lambda(x)=\varkappa \frac{T_{\mu \nu} M^{\mu \nu}}{M^{\alpha \beta} G_{\alpha \beta}},
$$

which yields

$$
T_{\mu \nu}=\frac{T_{\sigma \lambda} M^{\sigma \lambda}}{M^{\alpha \beta} G_{\alpha \beta}} G_{\mu \nu} .
$$

In particular, one could even use inverse tensors $\left(T^{-1}\right)^{\mu \nu}$ or $\left(G^{-1}\right)^{\mu \nu}$ or $\left(R^{-1}\right)^{\mu \nu}$ : defined as $\left(T^{-1}\right)^{\mu \lambda} T_{\lambda \nu}=\delta_{\nu}^{\mu}$ etc., provided they exist ${ }^{10}$. In this case, one would write

$$
G_{\mu \nu}=\frac{G_{\sigma \lambda}\left(T^{-1}\right)^{\lambda \sigma}}{4} T_{\mu \nu},
$$

instead of (28).

\section{EINSTEIN-TRANSVERSALITY IN ACTION FOR SCALE-FREE GRAVITY}

By analogy with the Henneaux-Teitelboim formulation of the unimodular gravity (15), it is easy to write similar

\footnotetext{
${ }^{10}$ For a very recent discussion of other use of inverse Ricci tensor
} or anti-curvature in cosmology, see [60]. actions for a global degree of freedom representing the Newton constant:

$$
S[g, C, \alpha]=\frac{1}{2} \int d^{4} x \sqrt{-g}\left(\nabla_{\mu} C^{\mu}-R\right) \alpha .
$$

An action similar to (54) was used in [61,62] for local version of the vacuum energy sequester [63]. On the other hand, following (25) one can exchange the vector $C^{\mu}$ with the Chern-Simons current of a gauge field $\mathcal{A}_{\mu}$ and write

$$
S[g, \mathcal{A}, \alpha]=\frac{1}{2} \int d^{4} x \sqrt{-g}\left(\mathcal{F}_{\mu \nu} \mathcal{F}^{\star \mu \nu}-R\right) \alpha .
$$

Here we assume standard general-covariant and minimally coupled action for all usual matter fields.

Checking the equations of motion for (54) we have

$$
\frac{2}{\sqrt{-g}} \cdot \frac{\delta S}{\delta C^{\mu}}=-\partial_{\mu} \alpha=0,
$$

and for the metric variation

$$
\begin{aligned}
& \delta_{g} S=\frac{1}{2} \int d^{4} x\left[-\alpha \delta_{g}(\sqrt{-g} R)+\alpha \delta_{g} \partial_{\mu}\left(\sqrt{-g} C^{\mu}\right)\right]= \\
& =\frac{1}{2} \int d^{4} x\left[-\alpha \delta_{g}(\sqrt{-g} R)-(\delta \sqrt{-g}) C^{\mu} \partial_{\mu} \alpha\right],
\end{aligned}
$$

so that on equation of motion for $C^{\mu}$ the divergence term does not contribute to the metric equation of motion. In this way, for the standard action of the minimally coupled matter fields one obtains

$$
\alpha G_{\mu \nu}=T_{\mu \nu}
$$

and consequently

$$
\alpha=\frac{1}{8 \pi G_{N}} .
$$

On the other hand, $\sqrt{-g} \mathcal{F}_{\mu \nu} \mathcal{F}^{\star \mu \nu}$ does not contribute to the tensor equations of motion, see (26). Whereas for abelian $\mathcal{A}_{\beta}$

$$
\frac{2}{\sqrt{-g}} \cdot \frac{\delta S}{\delta \mathcal{A}_{\gamma}}=4 \mathcal{F}^{\star \gamma \mu} \partial_{\mu} \alpha=0 .
$$

However, to conclude here that $\partial_{\mu} \alpha=0$ one has to assume the existence of $\left(\mathcal{F}^{\star \gamma \mu}\right)^{-1}$. The Hodge dual of the field tensor has an inverse provided $\mathcal{F}_{\mu \nu} \mathcal{F}^{\star \mu \nu} \neq 0$, for a recent discussion see [64], hence one should assume that $R$ is not identically vanishing ${ }^{11}$.

The canonical structure is more transparent for the action (54). There one can either follow a direct analogy with the Henneaux and Teitelboim formulation of the unimodular gravity [22], and Faddeev-Jackiw [27, 28]

\footnotetext{
${ }^{11}$ It is worth noting here again that in the early universe $R$ is not vanishing [59].
} 
procedure or look at the Hamiltonian analysis performed for the vacuum energy sequester, see [65-67]. Similarly to (16), in this action $\alpha$ is a canonical momentum conjugated to the global quantity

$$
\mathscr{R}(t)=\frac{1}{2} \int_{\Sigma} d^{3} \mathbf{x} \sqrt{-g} C^{t}(t, \mathbf{x}),
$$

which measures integrated Ricci scalar between the Cauchy hypersurfaces $\Sigma_{2}$ and $\Sigma_{1}$

$$
\mathscr{R}\left(t_{2}\right)-\mathscr{R}\left(t_{1}\right)=\frac{1}{2} \int_{\mathscr{V}} d^{4} x \sqrt{-g} R .
$$

Again one can think about $\mathscr{R}$ as a charge which is not conserved, but rather continuously produced by Ricci curvature which plays the role of a source

$$
\nabla_{\mu} C^{\mu}=R
$$

Thus, the inverse Newton constant $\alpha$ is canonically conjugated $^{12}$ to the spacetime averaged Ricci curvature $\mathscr{R}(t)$. On the other hand, $\mathscr{R}$ is clearly proportional to the Einstein-Hilbert (volume) part of the gravitational action. Thus, this resembles action-angle variables. As $\alpha$ is canonically conjugated to $\mathscr{R}$ one can directly apply the Heisenberg uncertainty relation to obtain

$$
\frac{\delta G_{N}}{G_{N}} \times \frac{\delta \int_{\mathscr{V}} d^{4} x \sqrt{-g} R}{\ell_{P l}^{2}} \geq 8 \pi .
$$

It is instructive to express this inequality in terms of fluctuations of the corresponding Planck length $\ell_{P l}=\sqrt{\hbar G_{N}}$

$$
\frac{\delta \ell_{P l}}{\ell_{P l}} \times \frac{\delta \int_{\mathscr{V}} d^{4} x \sqrt{-g} R}{\ell_{P l}^{2}} \geq 4 \pi .
$$

Similarly to (19), these inequalities may have nontrivial consequences close to singularities. Interestingly, $\hbar$ is not explicit in these uncertainty relations above.

Following discussion around equations (22), (23) and (24) and applying them to $C^{\mu}$ instead of $W^{\mu}$ one can try to fix

$$
C^{\mu}=\delta_{t}^{\mu} \frac{t}{\sqrt{-g}} M_{\alpha}^{2}
$$

in the action, before variation. Here $M_{\alpha}$ is some mass scale introduced for dimensional reasons. Then, in units where $M_{\alpha}=1$, the action takes a non-covariant form

$$
S[g, \alpha]=\frac{1}{2} \int d^{4} x(1-\sqrt{-g} R) \alpha,
$$

thus instead of the "unimodular" constraint (24) we have

$$
\sqrt{-g} R=1
$$

\footnotetext{
${ }^{12}$ For thermodynamical arguments in favor of this relation, see
} $[68,69]$. or the "unicurvature" condition. Similarly to what is often done in the unimodular gravity one can forget about gauge and coordinate fixing steps resulting in (66) and take this non-covariant action (67), as a starting point.

Following a nice discussion in [26], one can always construct such coordinates where (68) is fulfilled. Suppose in some coordinates $\left(t^{\prime}, x^{\prime i}\right)$ we have $\sqrt{-g^{\prime}} R^{\prime} \neq 1$. Then using that the Ricci density transforms as

$$
\sqrt{-g^{\prime}} R^{\prime}=\left|\frac{\partial x}{\partial x^{\prime}}\right| \sqrt{-g} R
$$

we can find such coordinates $\left(t, x^{i}\right)$ that $x^{i}=x^{i}$, but

$$
t=\int^{t^{\prime}} d t^{\prime \prime} \sqrt{-g^{\prime}\left(t^{\prime \prime}, x^{\prime i}\right)} R^{\prime}\left(t^{\prime \prime}, x^{i}\right),
$$

so that one obtains

$$
\left|\frac{\partial x}{\partial x^{\prime}}\right|=\frac{\partial t}{\partial t^{\prime}}=\sqrt{-g^{\prime}} R^{\prime}
$$

which enforces the "unicurvature" condition (68) in coordinates $\left(t, x^{i}\right)$. However, similarly to the non-covariant formulation of unimodular gravity one cannot just find coordinates where both conditions (66) and (68) hold simultaneously. Therefore the latter condition is an equation of motion implying nontrivial dynamics.

Let us check that, despite the illegitimate step of fixing the gauge directly in the action before variation, still the action (67) reproduces the same dynamics as the original general-covariant action (54). Variation with respect to $\delta g^{\mu \nu}$ of (67) accompanied with the action for minimally coupled matter yields

$$
\alpha G_{\mu \nu}-\nabla_{\mu} \nabla_{\nu} \alpha+g_{\mu \nu} \square \alpha=T_{\mu \nu},
$$

where $\square=g^{\mu \nu} \nabla_{\mu} \nabla_{\nu}$. Here we are obviously missing (56) which enforces that $\alpha$ is a constant. However, applying $\nabla^{\mu}$ to both sides of the equation (72) one obtains

$$
G_{\mu \nu} \nabla^{\mu} \alpha+\nabla_{\nu} \square \alpha-\square \nabla_{\nu} \alpha=0,
$$

where we used the Bianchi identity and EMT conservation. Further, invoking the identity

$$
\nabla_{\nu} \square \alpha-\square \nabla_{\nu} \alpha=-R_{\mu \nu} \nabla^{\mu} \alpha,
$$

yields $R \partial_{\nu} \alpha=0$, so that we are back to (58).

Interestingly, varying the "unicurvature" constraint (68) one obtains

$$
G_{\mu \nu} \bar{\delta} g^{\mu \nu}=\nabla_{\mu} \nabla_{\nu} \bar{\delta} g^{\mu \nu}-g_{\mu \nu} \square \bar{\delta} g^{\mu \nu}
$$

reproducing the "Einstein transversality" condition (34) only up to a divergence.

It is worth mentioning, that similarly to what is sometimes done in the unimodular gravity one can also substitute $\sqrt{-g}=R^{-1}$ into matter part of the action. This would produce an apparently new and crazily looking theory without changing any physics. 
Finally, we would like to mention that it is the absence of the usual kinetic term $\mathcal{F}_{\mu \nu} \mathcal{F}^{\mu \nu}$ which forces $\alpha$ to be constant. Hence, similarly to (27), one can write

$$
\begin{aligned}
& S[g, \mathcal{A}, \nu]=\int d^{4} x \sqrt{-g}\left[-\frac{1}{2} \nu^{2} R+\right. \\
& \left.+\frac{1}{2}(\partial \nu)^{2}+\frac{\nu}{f_{\alpha}} \mathcal{F}_{\gamma \sigma} \mathcal{F}^{\star \gamma \sigma}-V_{\alpha}(\nu)\right],
\end{aligned}
$$

where now $\nu$ is canonically normalized pseudoscalar and $f_{\alpha}$ is some mass scale emulating the axion would-be decay constant, while $V_{\alpha}(\nu)$ is a potential. Again, the presence of the standard kinetic term for $\nu$ does not change anything, as on-shell $\nu$ always remains an arbitrary constant $\nu_{\star}$. In particular, the kinetic term may be non-canonical. Another advantage is that this action has usual properties regarding parity transformations. The action is written in the Jordan frame. The potential induces cosmological constant $V_{\alpha}\left(\nu_{\star}\right)$ whose presence is also useful for invertibility of $\mathcal{F}^{\star \alpha \beta}$. This construction gives hope to find the scale-free gravity (28) as a particular dynamical regime (maybe strongly coupled) of a more usual QFT system.

\section{ENERGY-TRANSVERSALITY IN ACTION FOR SCALE-FREE GRAVITY}

On the other hand, one can attain the effective Newton constant as a global degree of freedom by changing the usual action for matter fields $\Phi_{m}$,

$$
S_{0}\left[g, \Phi_{m}\right]=\int d^{4} x \sqrt{-g} \mathscr{L}_{m}
$$

to the one (c.f. [70]) similar to the Henneaux and Teitelboim construction (15)

$$
S\left[g, \beta, L, \Phi_{m}\right]=\int d^{4} x \sqrt{-g} \beta\left(\mathscr{L}_{m}-\nabla_{\lambda} L^{\lambda}\right)
$$

Or instead of the vector field $L^{\lambda}$ one can again use a gauge field $\mathscr{A}_{\mu}$

$$
S\left[g, \beta, \mathscr{A}, \Phi_{m}\right]=\int d^{4} x \sqrt{-g} \beta\left(\mathscr{L}_{m}-\mathscr{F}_{\mu \nu} \mathscr{F}^{\star \mu \nu}\right),
$$

so that the "axionic" field $\beta$ universally couples to all matter fields. Here we assume that the gravitational sector is described by the standard Einstein-Hilbert action. We could slightly modify the action in order to have better transformation properties with respect to parity symmetry and write

$$
S\left[g, \eta, \mathscr{A}, \Phi_{m}\right]=\int d^{4} x \sqrt{-g} \eta\left(\eta \mathscr{L}_{m}-\mathscr{F}_{\mu \nu} \mathscr{F}^{\star \mu \nu}\right) .
$$

Under parity $\eta$ (and $\beta$ ) is a pseudoscalar which transforms as $\eta \rightarrow-\eta$. Lest the normal matter become ghosty under parity transformation, one should use this improved version of the action (80).

Of course, completely analogously to previous cases, the variation with respect to the vector field $L^{\mu}$ (or $\mathscr{A}_{\mu}$ ) results in $\partial_{\mu} \beta=0$ (or $\partial_{\mu} \eta=0$ ). On shell $\eta=\eta_{\star}$, which opens a path to further modification of this action to make it look more familiar

$$
\begin{aligned}
S\left[g, \eta, \mathscr{A}, \Phi_{m}\right] & =\int d^{4} x \sqrt{-g}\left[\frac{1}{2}(\partial \eta)^{2}-V_{\beta}(\eta)+\right. \\
& \left.+\frac{\eta^{2}}{M_{m}^{2}} \mathscr{L}_{m}-\frac{\eta}{f_{\beta}} \mathscr{F}_{\mu \nu} \mathscr{F}^{\star \mu \nu}\right]
\end{aligned}
$$

where $V_{\beta}(\eta)$ is a potential and the usual dimensions of $\eta$ are restored by introduction of two mass scales $M_{m}$ and $f_{\beta}$. And again the presence of the potential introduces a cosmological constant $V_{\beta}\left(\eta_{\star}\right)$. This guarantees the existence of $\left(\mathscr{F}^{\star \mu \nu}\right)^{-1}$ required to infer the constancy of $\eta$. It is crucial that the axion $\eta$ is universally coupled to all other matter fields. While gravity is assumed to be described by the standard Einstein-Hilbert action so that the description is in the Einstein frame. Clearly, in our setup the mass scales and even the form of the potential $V_{\beta}(\eta)$ and the form of the kinetic term (which can be again non-canonical) are rather irrelevant. However, in any attempt to embed this theory into more usual QFT setup these will be crucial bits of information. This action appears from standard Yang-Mills construction provided one takes the limit when one can neglect the usual kinetic term $-\mathscr{F}_{\mu \nu} \mathscr{F}^{\mu \nu} / 4 \mathrm{~g}^{2}$ for the gauge field. Naively, this limit corresponds to a confinement or an infinitely strong coupling $\mathrm{g} \rightarrow \infty$.

It is worth noting that combining action (81) or (76) with the (27) provides an axionic formulation of the vacuum energy sequestering $[4,61,63]$. This novel axionic formulation of sequester is much closer to the usual particle physics models.

The canonical structure of the system is more transparent for the action (78). In this formulation of the theory, on the right hand side of the Einstein equations one obtains a rescaled stress tensor for matter fields

$$
T_{\mu \nu}=\frac{2}{\sqrt{-g}} \frac{\delta S}{\delta g^{\mu \nu}}=\beta T_{\mu \nu}^{(m)},
$$

where

$$
T_{\mu \nu}^{(m)}=\frac{2}{\sqrt{-g}} \frac{\delta S_{0}}{\delta g^{\mu \nu}}=\frac{2}{\sqrt{-g}} \frac{\delta\left(\sqrt{-g} \mathscr{L}_{m}\right)}{\delta g^{\mu \nu}},
$$

is defined exclusively through the usual Lagrangian density or from the original action (77). In the last equality we assumed that matter sector is standard and in particular that the Lagrangian does not depend on the derivatives of the metric. In fact, this last equality would not be applicable for Kinetic Gravity Braiding [71] and G-Inflation [72].

Similarly to (16) and (61) the dynamical degree of freedom canonically conjugated to $\beta$ is

$$
I(t)=-\int_{\Sigma} d^{3} \mathbf{x} \sqrt{-g} L^{t}(t, \mathbf{x})
$$


which measures the usual matter action between the Cauchy hypersurfaces $\Sigma_{2}$ and $\Sigma_{1}$

$$
I\left(t_{2}\right)-I\left(t_{1}\right)=-\int_{\mathscr{V}} d^{4} x \sqrt{-g} \mathscr{L}_{m} .
$$

Also here one can think about $I(t)$ as a charge which is not conserved, but rather continuously produced by the total matter Lagrangian which plays the role of a source

$$
\nabla_{\mu} L^{\mu}=\mathscr{L}_{m} .
$$

On the other hand, the introduction of $\beta$ rescales the commutation relations for usual matter. In the same way, in the approach with the Einstein-transverse condition described in (V) a similar rescaling (89) with $1 / G_{N}$ instead of $\beta$ occurs for the commutator of the dimensionless spatial metric and it's conjugated momentum given by the trace-free extrinsic curvature density, see [47].

For example, for the usual scalar field $\phi$ the canonical momentum gets rescaled in the analogous way to the EMT:

$$
\pi=\beta \pi^{(m)}=\beta \sqrt{-g} \frac{\partial \mathscr{L}_{m}}{\partial \dot{\phi}} .
$$

Hence, if the canonical commutator is

$$
[\phi(\mathbf{x}), \pi(\mathbf{y})]=i \hbar \delta(\mathbf{x}-\mathbf{y}),
$$

the usual commutator gets rescaled as

$$
\left[\phi(\mathbf{x}), \pi^{(m)}(\mathbf{y})\right]=\frac{i \hbar}{\beta} \delta(\mathbf{x}-\mathbf{y}),
$$

so that the effective Planck constant is

$$
\bar{\hbar}=\frac{\hbar}{\beta} .
$$

This is ideologically similar to what is discussed in [70], though our frameworks are different.

For the usual Einstein-Hilbert action with fixed $G_{N}$ one obtains rescaled Einstein equations

$$
\frac{G_{\mu \nu}}{8 \pi G_{N}}=\beta T_{\mu \nu}^{(m)}
$$

so that the effective Newton constant, $\bar{G}_{N}$, is rescaled as

$$
\bar{G}_{N}=G_{N} \beta .
$$

Interestingly, one obtains

$$
\bar{\hbar} \bar{G}_{N}=\hbar G_{N},
$$

so that the Planck length (and time) $\ell_{P l}=\sqrt{\hbar G_{N}}$ remain invariant. This discussion is applicable if the universe is in an eigenstate of $\beta$.

On the other hand, in generic state, $\beta$ can have its own quantum fluctuations. In that case, we again write the
Heisenberg uncertainty relation for the fluctuation of the effective Newton constant (92)

$$
\delta \bar{G}_{N} \times \delta \int_{\mathscr{V}} d^{4} x \sqrt{-g} \mathscr{L}_{m} \geq \frac{1}{2} \ell_{P l}^{2},
$$

or equivalently for the effective Planck constant (90)

$$
\delta \bar{\hbar} \times \delta \int_{\mathscr{V}} d^{4} x \sqrt{-g} \mathscr{L}_{m} \geq \frac{1}{2} \bar{\hbar}^{2} .
$$

We should remark that in case of the action (80) instead of $\beta$ one should use $\eta^{2}$ in all rescaling above, while for (81) one uses $\eta^{2} / M_{m}^{2}$.

Interestingly, the invariance of $\ell_{P l}$ given by (93) does not allow for fluctuations of $\ell_{P l}$, contrary to those (65) present in the approach with the Einstein-transverse condition.

One can also combine both actions (78) and (54) and obtain the Einstein equations written as

$$
\alpha G_{\mu \nu}=\beta T_{\mu \nu}^{(m)},
$$

so that the effective Newton constant is

$$
\bar{G}_{N}=\frac{1}{8 \pi} \frac{\beta}{\alpha} .
$$

\section{A. "Noncovariant" or "unimatter" formulation.}

Finally, similarly to fixing $W^{\mu}$ as in Eq. (24) for the non-covariant formulation of the unimodular gravity and to fixing $C^{\mu}$ as in Eq. (66) for the non-covariant "unicurvature" gravity, one can try to illegitimately fix

$$
L^{\mu}=\delta_{t}^{\mu} \frac{t}{\sqrt{-g}} M_{\beta}^{4},
$$

before varying the action (78). Here $M_{\beta}$ is some mass scale. Then, again in units where this mass scale is unity, $M_{\beta}=1$, the action takes an unusual non-covariant form

$$
S\left[g, \beta, \Phi_{m}\right]=\int d^{4} x \beta\left(\sqrt{-g} \mathscr{L}_{m}-1\right),
$$

implying unity of the matter Lagrangian density, which is clearly different from Eq. (4) from [70]. On top, we again assume the usual Einstein-Hilbert action with fixed $G_{N}$. One could call this "unimatter gravity", as

$$
\sqrt{-g} \mathscr{L}_{m}=1,
$$

which corresponds to a coordinate choice or a partial gauge fixing, modulo the point which we have already discussed after Eq. (24). We can again repeat the same procedure to find proper coordinates where "unimatter" condition holds by applying formulas (69), (70) and (71) with exchanging $R$ with $\mathscr{L}_{m}$.

From the usual definition of the EMT (83) we have

$$
\delta\left(\sqrt{-g} \mathscr{L}_{m}\right)=\frac{\sqrt{-g}}{2} T_{\mu \nu}^{(m)} \bar{\delta} g^{\mu \nu}=0,
$$


thus the "unimatter" condition (100) reproduces the "energy transversality" condition (35) restricting the variations $\bar{\delta} g^{\mu \nu}$.

If, as it is the case for the Standard Model fields, $\mathscr{L}_{m}$ does not contain derivatives of the metric, then the variation with respect to the metric yields (91). However, contrary to the general-covariant formulation, now $\beta(x)$ is a free function similarly to $(36)$, as we do not have an equation of motion for $L^{\mu}$ to impose $\partial_{\mu} \beta=0$. In our restricted variational formulation with energytransversality condition (35) it was the joint work of the Bianchi identity and the assumed conservation of the EMT which resulted in $\partial_{\mu} \beta=0$. However, similarly to what happens [52] (c.f. [38]) in the non-covariant formulation (24) of the unimodular gravity, due to the noncovariant formulation one cannot just assume the conservation of $T_{\mu \nu}^{(m)}$.

To illustrate this point, lets first consider just one scalar field $\phi$, as general as k-essence $[73,74]$. This can be also considered as a proxy for irrotational hydrodynamics. The Euler-Lagrange equation for $\phi$ from the action (99) is

$$
\sqrt{-g} \beta \frac{\partial \mathscr{L}_{m}}{\partial \phi}=\partial_{\mu}\left(\sqrt{-g} \beta \frac{\partial \mathscr{L}_{m}}{\partial \partial_{\mu} \phi}\right)
$$

and can be rewritten as

$$
\nabla_{\mu}\left(\frac{\partial \mathscr{L}_{m}}{\partial \partial_{\mu} \phi}\right)-\frac{\partial \mathscr{L}_{m}}{\partial \phi}=-\frac{\partial \mathscr{L}_{m}}{\partial \partial_{\mu} \phi} \frac{\partial_{\mu} \beta}{\beta} .
$$

Further, for the scalar field the Noether form of the EMT is already symmetric and can be written as

$$
T_{\beta}^{\alpha(m)}=\frac{\partial \mathscr{L}_{m}}{\partial \partial_{\alpha} \phi} \partial_{\beta} \phi-\mathscr{L}_{m} \delta_{\beta}^{\alpha},
$$

while its divergence is

$$
\nabla_{\alpha} T_{\beta}^{\alpha(m)}=\left(\nabla_{\alpha}\left(\frac{\partial \mathscr{L}_{m}}{\partial \partial_{\alpha} \phi}\right)-\frac{\partial \mathscr{L}_{m}}{\partial \phi}\right) \partial_{\beta} \phi
$$

In the usual case, the expression in front of $\partial_{\beta} \phi$ would be the equation of motion so that the EMT would be conserved. However, here the equation of motion (103) is modified by the presence of $\beta$ so that there is a nonconservation:

$$
\nabla_{\alpha} T_{\mu}^{\alpha(m)}=-\frac{\partial \mathscr{L}_{m}}{\partial \partial_{\alpha} \phi} \frac{\partial_{\alpha} \beta}{\beta} \partial_{\mu} \phi .
$$

Now we can apply covariant derivative to the Einstein equations (91) and obtain

$$
\begin{aligned}
& \frac{\nabla_{\alpha} G_{\mu}^{\alpha}}{8 \pi G_{\mathrm{N}}}=\nabla_{\alpha}\left(\beta T_{\mu}^{\alpha(m)}\right)= \\
& =T_{\mu}^{\alpha(m)} \partial_{\alpha} \beta+\beta \nabla_{\alpha} T_{\mu}^{\alpha(m)}=-\mathscr{L}_{m} \partial_{\mu} \beta,
\end{aligned}
$$

where we used (106) and (104). Hence, the Bianchi identity, indeed, again implies that $\beta$ is a constant. This, of course, also results in the conservation of $T_{\mu \nu}^{(m)}$.
Now let us consider usual free electrodynamics ${ }^{13}$

$$
\mathscr{L}_{m}=-\frac{1}{4} F_{\mu \nu} F^{\mu \nu}+\Lambda
$$

so that

$$
\frac{\partial \mathscr{L}_{m}}{\partial \partial_{\mu} A_{\nu}}=-F^{\mu \nu}
$$

and we have the same equations of motion (103), where just instead of $\phi$ we have $A_{\mu}$, so that

$$
\nabla_{\lambda} F^{\mu \lambda}=-F^{\mu \lambda} \frac{\partial_{\lambda} \beta}{\beta} .
$$

The symmetric Belinfante-Rosenfeld / Hilbert EMT relevant for gravity

$$
T_{\nu}^{\mu(m)}=F_{\lambda}^{\mu} F_{\nu}^{\lambda}-\mathscr{L}_{m} \delta_{\nu}^{\mu},
$$

deviates from the canonical Noether one (104) which is not even covariantly conserved in the presence of gravity. In the absence of the electromagnetic current one obtains

$$
\nabla^{\mu} T_{\mu \nu}^{(m)}=-F_{\lambda \nu} \nabla_{\mu} F^{\lambda \mu}=F_{\lambda \nu} F^{\lambda \alpha} \frac{\partial_{\alpha} \beta}{\beta} .
$$

Substituting this expression for non-conservation of EMT (112) along with (111) into the left hand side of (107) we again obtain the last equality there which, in turn, implies $\beta=$ const.

In both examples above we essentially used explicit forms (104) and (111) of the EMT. The case with the scalar field is easily generalizable to many scalar fields, but this is not a generic matter source. Unfortunately, for generic matter with internal spin, the canonical Noether EMT is not symmetric and is not a source of gravity and does not coincide with the proper Hilbert EMT obtained through the variation of the action with respect to the metric. Below we provide the proof of the last equality in (107) for generic usual matter, whose Lagrangian does not involve derivatives of the metric. However, this proof does not cover fermions, as we do not want to overcomplicate the discussion by involving tetrads.

The "unimatter" action (99) is written in a particular class of coordinates where (98) holds. Let us denote one particular system of such coordinates $X^{\mu}$. Now we can write this action (99) in arbitrary coordinates $y^{\mu}$ by noting that $\mathscr{L}_{m}, d^{4} x \sqrt{-g}$ and $\beta$ are scalar objects:

$$
S_{X}\left[g, \beta, \Phi_{m}\right]=\int d^{4} y \beta\left(\sqrt{-g} \mathscr{L}_{m}-\left|\frac{\partial X}{\partial y}\right|\right),
$$

where $|\partial X / \partial y|$ is the Jacobian. We should stress that in this action the original coordinates $X^{\mu}(y)$ are not

13 supplemented by a cosmological constant $\Lambda$ to avoid that $\mathscr{L}_{m}=0$ on electromagnetic waves which are solutions of the free electrodynamics. 
dynamical variables and remain "frozen" like external sources or background fields. One could promote them to four Stückelberg scalar fields similarly to how it is done for the unimodular gravity in [23], but we refrain from doing this. Further we can recall that the measure transforms as

$$
d^{4} X \sqrt{-g(X)}=d^{4} y\left|\frac{\partial X}{\partial y}\right| \sqrt{-g(X)}=d^{4} y \sqrt{-g(y)},
$$

so that

$$
\left|\frac{\partial X}{\partial y}\right|=\frac{\sqrt{-g(y)}}{\sqrt{-g(X)}} .
$$

In this case the action (99) assumes the generally covariant form

$$
S_{X}\left[g, \beta, \Phi_{m}\right]=\int d^{4} y \sqrt{-g} \beta\left(\mathscr{L}_{m}-\frac{1}{\sqrt{-g(X)}}\right),
$$

because with respect to further coordinate transformations $y \rightarrow y\left(y^{\prime}\right)$ the background field $\sqrt{-g(X)}$ transforms as a scalar. Now we can consider an infinitesimal change of coordinates

$$
y^{\prime \mu}=y^{\mu}-\xi^{\mu}(y)
$$

which leads to the variations given by the Lie derivatives

$$
\begin{gathered}
£_{\xi}(-g(X))^{-1 / 2}=\xi^{\mu} \partial_{\mu}(-g(X))^{-1 / 2}, \\
£_{\xi} g^{\mu \nu}=-\xi^{\mu ; \nu}-\xi^{\nu ; \mu}, \\
£_{\xi}\left(\sqrt{-g} \mathscr{L}_{m}\right)=-\frac{\sqrt{-g}}{2} T_{\mu \nu}^{(m)}\left(\xi^{\mu ; \nu}+\xi^{\nu ; \mu}\right), \\
£_{\xi} \sqrt{-g}=\frac{1}{2} \sqrt{-g} g_{\mu \nu}\left(\xi^{\mu ; \nu}+\xi^{\nu ; \mu}\right),
\end{gathered}
$$

where ()$_{; \mu}=\nabla_{\mu}()$. We do not need variations of $\beta$ and of $\Phi_{m}$, as we assume that their equations of motion hold and result in vanishing variations of the action. Moreover, at this step we can use equation of motion for $\beta:(-g(X))^{-1 / 2}=\mathscr{L}_{m}$. With respect to the diffeomorphisms (117) the action remains invariant so that

$$
\int d^{4} y \sqrt{-g} \beta\left[-T_{\mu \nu}^{(m)} \xi^{\mu ; \nu}-\mathscr{L}_{m} g_{\mu \nu} \xi^{\mu ; \nu}-\xi^{\mu} \partial_{\mu} \mathscr{L}_{m}\right]=0 .
$$

Now we can integrate by parts the first two terms, what, under the assumption that $\xi^{\mu}$ vanishes on the boundary, provides

$$
\int d^{4} y \sqrt{-g}\left[\nabla^{\nu}\left(\beta T_{\mu \nu}^{(m)}\right)+\partial_{\mu}\left(\beta \mathscr{L}_{m}\right)-\beta \partial_{\mu} \mathscr{L}_{m}\right] \xi^{\mu}=0 .
$$

Taking into account arbitrariness of $\xi^{\mu}$ this expression yields

$$
\nabla^{\nu}\left(\beta T_{\mu \nu}^{(m)}\right)=-\mathscr{L}_{m} \partial_{\mu} \beta
$$

which should hold on all equations of motion. Hence, we again obtained last equality in (107) which now holds for generic matter with metric EMT.

Here we should mention that one could substitute the "unimatter" constraint (100) directly into the EinsteinHilbert action, similarly to what is sometimes done with the unimodular constraint. However, contrary to the latter case this does not enforces a polynomial form of this action in the metric.

\section{EINSTEIN FRAME}

As we have seen in the variational formulation "Einstein transversality" condition was equivalent to the "Energy transversality" condition. In the integral formulation of the "Einstein transversality" we assumed that the matter is minimally coupled. In these actions (54) and (55) one can make a Weyl transformation

$$
g_{\mu \nu}=e^{2 \omega} \gamma_{\mu \nu}
$$

in order to pass to an Einstein frame. The Weyl transformation above will not change the form of the axionic term $\alpha \mathcal{F}_{\mu \nu} \mathcal{F}^{\star \mu \nu} \sqrt{-g}$, while, after a field redefinition (Weyl transformation) of $C^{\mu}$, the term $\alpha \nabla_{\mu} C^{\mu} \sqrt{-g}$ will preserve its form as well. However, as it is well known

$$
R(g)=e^{-2 \omega}\left(R(\gamma)-6 \square_{\gamma} \omega-6 \gamma^{\mu \nu} \partial_{\mu} \omega \partial_{\nu} \omega\right),
$$

where $\square_{\gamma}=\gamma^{\mu \nu} \widetilde{\nabla}_{\mu} \widetilde{\nabla}_{\nu}$ and $\widetilde{\nabla}_{\mu}$ is the Levi-Civita connection compatible with the transformed metric

$$
\widetilde{\nabla}_{\mu} \gamma_{\alpha \beta}=0
$$

Further, by taking

$$
\omega=-\frac{1}{2} \log \alpha
$$

and introducing

$$
\chi=\sqrt{6} \omega=-\sqrt{\frac{3}{2}} \log \alpha
$$

one obtains the action (55) in the Einstein frame

$$
\begin{aligned}
S[\gamma, \mathcal{A}, \chi] & =\int d^{4} x \sqrt{-\gamma}\left[-\frac{1}{2} R(\gamma)+\frac{1}{2} \gamma^{\mu \nu} \partial_{\mu} \chi \partial_{\nu} \chi+\right. \\
& \left.+e^{-\sqrt{2 / 3} \chi} \mathcal{F}_{\mu \nu} \mathcal{F}^{\star \mu \nu}\right] .
\end{aligned}
$$

The usual matter is now coupled to the metric

$$
g_{\mu \nu}=e^{2 \omega} \gamma_{\mu \nu}=e^{\sqrt{2 / 3} \chi} \gamma_{\mu \nu}
$$

Here we omitted boundary terms. Interestingly, the field $\chi$ which is just a mere field redefinition of the Lagrange multiplier $\alpha$, looks now as a healthy scalar field with a usual non-ghosty canonical kinetic term, though this 
term does not play any role as it is zero due to the vector equations of motion, similarly to what happens in the action (81).

Clearly, for the vector field action one would get

$$
\begin{aligned}
S[\gamma, \widetilde{C}, \chi] & =\int d^{4} x \sqrt{-\gamma}\left[-\frac{1}{2} R(\gamma)+\frac{1}{2} \gamma^{\mu \nu} \partial_{\mu} \chi \partial_{\nu} \chi+\right. \\
+ & \left.e^{-\sqrt{2 / 3} \chi} \widetilde{\nabla}_{\mu} \widetilde{C}^{\mu}\right]
\end{aligned}
$$

where the vector $C^{\mu}$ got transformed according to it's conformal weight 4 :

$$
\widetilde{C}^{\mu}=e^{4 \omega} C^{\mu}=\alpha^{-2} C^{\mu}
$$

Of course we could omit the kinetic term for $\chi$ in these Einstein frame actions. Then these actions deviate from the (78) and (79) only by matter fields rescaling. The rescaling of the matter metric is in accord with what was discussed in the vacuum sequester [61-63, 75-77].

\section{One constant of integration to fix both $G_{N}$ and $\Lambda$}

One can further extend (76) by writing

$$
\begin{aligned}
S[g, \mathcal{A}, \varphi]=\int d^{4} x \sqrt{-g} & {\left[-\frac{1}{2} \mu^{2}(\varphi) R+\alpha(\varphi) \mathcal{F}_{\mu \nu} \mathcal{F}^{\star \mu \nu}+\right.} \\
+ & \left.\frac{1}{2}(\partial \varphi)^{2}-V(\varphi)\right]
\end{aligned}
$$

where $\mu(\varphi), \alpha(\varphi)$ and $V(\varphi)$ are arbitrary functions of the scalar field $\varphi$ and the normal matter is minimally coupled. One should assume that $\varphi$ is a pseudoscalar so that $\alpha(-\varphi)=-\alpha(\varphi)$. The gauge field equation of motion (60) gets an irrelevant modification

$$
\frac{1}{\sqrt{-g}} \cdot \frac{\delta S}{\delta \mathcal{A}_{\gamma}}=4 \alpha^{\prime} \mathcal{F}^{\star \gamma \mu} \partial_{\mu} \varphi=0,
$$

where prime denotes derivative with respect to $\varphi$. This equation has an obvious solution

$$
\varphi=\varphi_{\star}=\text { const }
$$

While the variation with respect to $\varphi$ yields constraint

$$
\alpha^{\prime} \mathcal{F}_{\mu \nu} \mathcal{F}^{\star \mu \nu}-V^{\prime}=\mu \mu^{\prime} R .
$$

where we used (134). This constraint allows one to express the Pontryagin invariant, $\mathcal{F}_{\mu \nu} \mathcal{F}^{\star \mu \nu}$, through the Ricci scalar. The later is to obtain from the tensor equations of motion. The functional dependence $\mu(\varphi), \alpha(\varphi)$ reduces to a mere coefficient of proportionality while the "potential", $V(\varphi)$ only provides a linear shift, as all these functions are evaluated at $\varphi_{\star}$. This shift is important because it allows to have a nonvanishing Pontryagin invariant even for a vanishing Ricci scalar.
In this Jourdan frame one obtains that the effective Planck mass is given by

$$
M_{P l}=\frac{\mu\left(\varphi_{\star}\right)}{\sqrt{8 \pi}},
$$

while the potential provides the effective cosmological constant

$$
\Lambda=V\left(\varphi_{\star}\right)
$$

Thus one integration constant provides the values for both coupling constants of GR. Let us briefly discuss the functions which can appear.

Simplest option from dimensional reasons would be $\mu(\varphi)=\varphi$. If the gauge filed is abelian, it can have arbitrary dimensionality as we neglect the standard kinetic term anyway. In particular, $[\mathcal{A}]=\left[m^{1 / 2}\right]$ would allow for the simplest coupling $\alpha=\varphi$. On the other hand, the form of the covariant derivative $D_{\mu}=\partial_{\mu}+i \mathrm{~g} \mathcal{A}_{\mu}$ with the self-coupling constant $g$ dictates that either the coupling constant $\mathrm{g}$ is dimensionless and $[\mathcal{A}]=[\mathrm{m}]$ so that one has to introduce a scale $M$ into the axion coupling $\alpha=\varphi / M$, or that $[\mathcal{A}]=\left[m^{1 / 2}\right]$ and $[\mathrm{g}]=\left[m^{1 / 2}\right]$. Thus the non-abelian realization provides a scale which can be used in the potential. In the later case one could write the potential as

$$
V(\varphi)=\mathrm{g}^{8} f\left(\frac{\varphi}{\mathrm{g}^{2}}\right) .
$$

Appealing forms of the potential would resemble instanton contribution

$$
V_{1}(\varphi)=\frac{1}{4} \varphi^{4} \exp \left(-\frac{\varphi}{\mathrm{g}^{2}}\right)
$$

and

$$
V_{2}(\varphi)=\frac{1}{4} \mathrm{~g}^{8} \exp \left(-\frac{\varphi}{\mathrm{g}^{2}}\right) .
$$

It would be great if the appearance of the scale in the coupling constant g could be related to the scale of strong coupling or dimensional transmutation. The strong coupling may hint to an explanation of the absence of the usual kinetic term for the gauge field in the action (132). To obtain 120 orders of magnitude difference one would need to assume: for $V_{1}(\varphi)$ that $\mathrm{g}^{2} \simeq \varphi_{\star} / 277$, whereas for $V_{2}(\varphi)$ that $\mathrm{g}^{2} \simeq \varphi_{\star} / 254$.

Finally we would like to note that the sign of the kinetic term for $\varphi$ can also be negative. Moreover, one can write an arbitrary function $P\left((\partial \varphi)^{2}, \varphi\right)$. Similarly to [78] the functional dependence on kinetic term does not change anything here.

\section{Conclusions}

In this paper we proposed a new very simple scalefree Einstein equations (5) which promote the Newton 
constant to a global degree of freedom. In this way the trace part of the usual Einstein equations is exchanged to a useless identity $1=1$. This is similar to trace-free Einstein equations of the unimodular gravity where this identity is $0=0$. Then we discussed different equivalent formulations of these equations and their variational formulations. After that we introduced different generally covariant actions (54), (55), (78), (79), (81), (132) describing Newton constant as a global degree of freedom. From these actions, we derived and discussed novel non-covariant "unicurvature" (67) and "unimatter" (99) actions following the same ideology as many works on unimodular gravity. It is interesting whether such noncovariant formulations can be further meaningfully generalized, as it was done in the case of unimodular gravity in [48-50]. Some of our frameworks for the dynamical Newton constant (78), (79), (81) actually imply that the effective Planck constant $\hbar$ becomes a global degree of freedom, similarly to claims of [70] where a different system was discussed. We have also written the Heisenberg uncertainty relations (64), (94) for the effective Newton constant $G_{N}$; (19) for cosmological constant $\Lambda$; (95) for the Planck constant $\hbar$ and (65) for the Planck length $\ell_{P l}$. Most probably these relations can only be physically relevant close to singularities either at the very beginning of our universe or at the final stages of gravitational collapse inside black holes. We left any detailed discussion of these uncertainty relations for future work.

Intriguingly, the unimodular gravity naturally appears in the thermodynamic or emergent gravity setup [79-82]. There are already some hints $[68,69]$ that the scale-free gravity (5) with the Newton constant as a constant of integration, may also emerge in this way. It is very interesting to further investigate this option.

On the other hand, combining unimodular gravity with theories where the Newton constant is another global degree of freedom is the key element of the vacuum sequester proposal, [4, 61, 63]. A novelty with respect to vacuum sequester works $[61-63,70,75-77]$ is that following [25] we also use normal Yang-Mills gauge fields instead of more exotic three- and four-forms to specify a new measure for the action integral. Moreover, in our formulation the Lagrange multiplier fields from these setups look like axions and can even have normal kinetic terms, see actions (27), (76) and (81). For the Newton constant to be a global degree of freedom the axion should either non-minimally couple to curvature as in (76) or to couple universally to all matter fields, as in (81). To describe sequester one requires to have at least two different gauge fields and two different axions. It is rather intriguing to understand quantum properties of such systems and their potential UV completions. In particular, such formulation can be also useful for supersymmetric generalizations, see e.g. [83-85]. Even though QCD seems to possess a composite three-form [56, 86-89], we think it is more convenient to see usual Yang-Mills gauge fields in the action. This brings the field content of such theories closer to elementary fields present already in the Standard Model. This may be a hint that it is the IR dynamics of the Yang-Mills vacuum (maybe even QCD or some "dark" QCD) and its topological properties which secretly define both gravitational constants $G_{N}$ and $\Lambda$.

\section{Acknowledgments}

It is a pleasure to thank I. Saltas and I. Sawicki, for valuable discussions and criticisms, while we are indebted to N.Kaloper and T. Padilla for very useful email exchange. K.S. would like to thank the Central European Institute for Cosmology and Fundamental Physics for their warm and welcoming hospitality during early stages of the project. K.S. is supported by JSPS KAKENHI Grant Number JP20J12585. M. Y. is supported in part by JSPS Grant-in-Aid for Scientific Research Numbers 18K18764 and JSPS Bilateral Open Partnership Joint Research Projects. This project originated when P. J. and A. V. were enjoying very warm hospitality of the cosmology group at the Tokyo Institute of Technology. This productive visit was possible thanks to the JSPS Invitational Fellowships for Research in Japan (Fellowship ID:S19062) received by A. V. The work of P. J. is supported by the Grant Agency of the Czech Republic GAČR grant 20-28525S. The work of A. V. is supported by the J.E. Purkyně Fellowship of the Czech Academy of Sciences and by the funds from the European Regional Development Fund and the Czech Ministry of Education, Youth and Sports (MŠMT): Project CoGraDS CZ.02.1.01/0.0/0.0/15_003/0000437.
[1] Y. B. Zel'dovich, "The Cosmological constant and the theory of elementary particles," Sov. Phys. Usp. 11 (1968) 381-393.

[2] S. Weinberg, "The Cosmological Constant Problem," Rev. Mod. Phys. 61 (1989) 1-23.

[3] J. Martin, "Everything You Always Wanted To Know About The Cosmological Constant Problem (But Were Afraid To Ask)," Comptes Rendus Physique 13 (2012) 566-665, arXiv:1205.3365 [astro-ph.CO].

[4] A. Padilla, "Lectures on the Cosmological Constant
Problem," arXiv:1502.05296 [hep-th].

[5] A. Einstein, "Spielen Gravitationsfelder im Aufbau der materiellen Elementarteilchen eine wesentliche Rolle?," Sitzungsber. Preuss. Akad. Wiss. Berlin (Math. Phys.) 1919 (1919) 349-356. https://einsteinpapers.press.princeton.edu/vol7-doc/178.

[6] J. Anderson and D. Finkelstein, "Cosmological constant and fundamental length," Am. J. Phys. 39 (1971) 901-904.

[7] W. G. Unruh, "A Unimodular Theory of Canonical 
Quantum Gravity," Phys. Rev. D40 (1989) 1048.

[8] W. G. Unruh and R. M. Wald, "Time and the Interpretation of Canonical Quantum Gravity," Phys. Rev. D40 (1989) 2598.

[9] D. R. Finkelstein, A. A. Galiautdinov, and J. E. Baugh, "Unimodular relativity and cosmological constant," $J$. Math. Phys. 42 (2001) 340-346, arXiv:gr-qc/0009099.

[10] G. F. R. Ellis, H. van Elst, J. Murugan, and J.-P. Uzan, "On the Trace-Free Einstein Equations as a Viable Alternative to General Relativity," Class. Quant. Grav. 28 (2011) 225007, arXiv:1008.1196 [gr-qc] .

[11] G. F. R. Ellis, "The Trace-Free Einstein Equations and inflation," Gen. Rel. Grav. 46 (2014) 1619, arXiv:1306.3021 [gr-qc].

[12] S. Alexander and R. Carballo-Rubio, "Topological Features of the Quantum Vacuum," Phys. Rev. D 101 no. 2, (2020) 024058, arXiv:1810.02159 [gr-qc].

[13] A. Einstein, "Letter to Michele Besso, August 20th,".

[14] C. O'Raifeartaigh, M. O'Keeffe, W. Nahm, and S. Mitton, "One hundred years of the cosmological constant: from "superfluous stunt" to dark energy," Eur. Phys. J. H 43 no. 1, (2018) 73-117, arXiv:1711.06890 [physics.hist-ph].

[15] A. Vilenkin, "The Birth of Inflationary Universes," Phys. Rev. D 27 (1983) 2848.

[16] A. D. Linde, "Eternally Existing Selfreproducing Chaotic Inflationary Universe," Phys. Lett. B 175 (1986) 395-400.

[17] A. D. Linde, "The Inflationary Universe," Rept. Prog. Phys. 47 (1984) 925-986.

[18] A. Linde, "A brief history of the multiverse," Rept. Prog. Phys. 80 no. 2, (2017) 022001, arXiv:1512.01203 [hep-th].

[19] S. Alexander, M. Cortês, A. R. Liddle, J. a. Magueijo, R. Sims, and L. Smolin, "Zero-parameter extension of general relativity with a varying cosmological constant," Phys. Rev. D 100 no. 8, (2019) 083506, arXiv:1905.10380 [gr-qc].

[20] S. Alexander, M. Cortês, A. R. Liddle, J. a. Magueijo, R. Sims, and L. Smolin, "Cosmology of minimal varying Lambda theories," Phys. Rev. D 100 no. 8, (2019) 083507, arXiv:1905.10382 [gr-qc].

[21] J. a. Magueijo and T. Złośnik, "Parity violating Friedmann Universes," Phys. Rev. D 100 no. 8, (2019) 084036, arXiv:1908.05184 [gr-qc].

[22] M. Henneaux and C. Teitelboim, "The Cosmological Constant and General Covariance," Phys. Lett. B222 (1989) 195-199.

[23] K. V. Kuchar, "Does an unspecified cosmological constant solve the problem of time in quantum gravity?," Phys. Rev. D43 (1991) 3332-3344.

[24] P. Jiroušek and A. Vikman, "New Weyl-invariant vector-tensor theory for the cosmological constant," JCAP 1904 (2019) 004, arXiv:1811.09547 [gr-qc].

[25] K. Hammer, P. Jiroušek, and A. Vikman, "Axionic cosmological constant," arXiv:2001.03169 [gr-qc].

[26] J. J. van der Bij, H. van Dam, and Y. J. Ng, "The Exchange of Massless Spin Two Particles," Physica 116A (1982) 307-320.

[27] L. D. Faddeev and R. Jackiw, "Hamiltonian Reduction of Unconstrained and Constrained Systems," Phys. Rev. Lett. 60 (1988) 1692-1694.

[28] R. Jackiw, "(Constrained) quantization without tears," in Diverse topics in theoretical and mathematical physics, pp. 163-175. 1993. arXiv:hep-th/9306075 [hep-th]. [,367(1993)].

[29] E. Álvarez, "Can one tell Einstein's unimodular theory from Einstein's general relativity?," JHEP 03 (2005) 002, arXiv:hep-th/0501146 [hep-th].

[30] B. Fiol and J. Garriga, "Semiclassical Unimodular Gravity," JCAP 1008 (2010) 015, arXiv:0809.1371 [hep-th].

[31] L. Smolin, "The Quantization of unimodular gravity and the cosmological constant problems," Phys. Rev. D 80 (2009) 084003, arXiv:0904.4841 [hep-th].

[32] A. Eichhorn, "On unimodular quantum gravity," Class. Quant. Grav. 30 (2013) 115016, arXiv:1301.0879 [gr-qc] .

[33] I. D. Saltas, "UV structure of quantum unimodular gravity," Phys. Rev. D90 no. 12, (2014) 124052, arXiv:1410.6163 [hep-th].

467. Padilla and I. D. Saltas, "A note on classical and quantum unimodular gravity," Eur. Phys. J. C75 no. 11, (2015) 561, arXiv:1409.3573 [gr-qc].

[35] R. Bufalo, M. Oksanen, and A. Tureanu, "How unimodular gravity theories differ from general relativity at quantum level," Eur. Phys. J. C75 no. 10, (2015) 477, arXiv:1505.04978 [hep-th].

[36] E. Álvarez, S. González-Martín, M. Herrero-Valea, and C. P. Martín, "Quantum Corrections to Unimodular Gravity," JHEP 08 (2015) 078, arXiv:1505.01995 [hep-th].

[37] E. Álvarez, S. González-Martín, M. Herrero-Valea, and C. P. Martín, "Unimodular Gravity Redux," Phys. Rev. D 92 no. 6, (2015) 061502, arXiv:1505.00022 [hep-th].

[38] R. Percacci, "Unimodular quantum gravity and the cosmological constant," Found. Phys. 48 no. 10, (2018) 1364-1379, arXiv:1712.09903 [gr-qc].

[39] R. de León Ardón, N. Ohta, and R. Percacci, "Path integral of unimodular gravity," Phys. Rev. D 97 no. 2, (2018) 026007, arXiv:1710.02457 [gr-qc].

[40] M. Herrero-Valea, "What do gravitons say about (unimodular) gravity?," JHEP 12 (2018) 106, arXiv:1806.01869 [hep-th].

[41] M. Herrero-Valea and R. Santos-Garcia, "Non-minimal Tinges of Unimodular Gravity," JHEP 09 (2020) 041, arXiv:2006.06698 [hep-th].

[42] G. P. de Brito and A. D. Pereira, "Unimodular quantum gravity: Steps beyond perturbation theory," JHEP 09 (2020) 196, arXiv:2007.05589 [hep-th].

[43] E. I. Guendelman and A. B. Kaganovich, "The Principle of nongravitating vacuum energy and some of its consequences," Phys. Rev. D 53 (1996) 7020-7025, arXiv: gr-qc/9605026.

[44] E. I. Guendelman, "Scale invariance, new inflation and decaying lambda terms," Mod. Phys. Lett. A14 (1999) 1043-1052, arXiv:gr-qc/9901017 [gr-qc].

[45] E. I. Guendelman and A. B. Kaganovich, "Dynamical measure and field theory models free of the cosmological constant problem," Phys. Rev. D 60 (1999) 065004, arXiv: gr-qc/9905029.

[46] R. L. Arnowitt, S. Deser, and C. W. Misner, "The Dynamics of general relativity," Gen. Rel. Grav. 40 (2008) 1997-2027, arXiv:gr-qc/0405109 [gr-qc].

[47] E. Poisson, A Relativist's Toolkit, The Mathematics of Black-Hole Mechanics. Cambridge University Press, 
2004.

[48] A. O. Barvinsky and A. Y. Kamenshchik, "Darkness without dark matter and energy - generalized unimodular gravity," Phys. Lett. B 774 (2017) 59-63, arXiv: 1705.09470 [gr-qc].

[49] A. O. Barvinsky, N. Kolganov, A. Kurov, and D. Nesterov, "Dynamics of the generalized unimodular gravity theory," Phys. Rev. D 100 no. 2, (2019) 023542, arXiv:1903.09897 [hep-th].

[50] A. O. Barvinsky, N. Kolganov, and A. Vikman, "Generalized unimodular gravity as a new form of k-essence," Phys. Rev. D 103 no. 6, (2021) 064035, arXiv:2011.06521 [gr-qc].

[51] A. O. Barvinsky and N. Kolganov, "Inflation in generalized unimodular gravity," Phys. Rev. D 100 no. 12, (2019) 123510, arXiv:1908.05697 [gr-qc].

[52] W. Buchmuller and N. Dragon, "Einstein Gravity From Restricted Coordinate Invariance," Phys. Lett. B207 (1988) 292-294.

[53] W. Buchmuller and N. Dragon, "Gauge Fixing and the Cosmological Constant," Phys. Lett. B223 (1989) 313-317.

[54] E. Alvarez, D. Blas, J. Garriga, and E. Verdaguer, "Transverse Fierz-Pauli symmetry," Nucl. Phys. B756 (2006) 148-170, arXiv:hep-th/0606019 [hep-th].

[55] I. Kimpton and A. Padilla, "Cleaning up the cosmological constant," JHEP 12 (2012) 031, arXiv:1203.1040 [hep-th].

[56] A. Aurilia, H. Nicolai, and P. Townsend, "Hidden Constants: The Theta Parameter of QCD and the Cosmological Constant of $\mathrm{N}=8$ Supergravity," Nucl. Phys. B 176 (1980) 509-522.

[57] M. Henneaux and C. Teitelboim, "The Cosmological Constant as a Canonical Variable," Phys. Lett. B 143 (1984) 415-420.

[58] F. Wilczek, "Foundations and Working Pictures in Microphysical Cosmology," Phys. Rept. 104 (1984) 143.

[59] R. R. Caldwell and S. S. Gubser, "Brief history of curvature," Phys. Rev. D 87 no. 6, (2013) 063523, arXiv:1302.1201 [astro-ph.CO].

[60] L. Amendola, L. Giani, and G. Laverda, "Ricci-inverse gravity: a novel alternative gravity, its flaws, and how to cure them," Phys. Lett. B $\mathbf{8 1 1}$ (2020) 135923, arXiv:2006.04209 [astro-ph.CO].

[61] N. Kaloper, A. Padilla, D. Stefanyszyn, and G. Zahariade, "Manifestly Local Theory of Vacuum Energy Sequestering," Phys. Rev. Lett. 116 no. 5, (2016) 051302, arXiv:1505.01492 [hep-th].

[62] N. Kaloper, "Irrational Monodromies of Vacuum Energy," JHEP 11 (2019) 106, arXiv:1806.03308 [hep-th].

[63] N. Kaloper and A. Padilla, "Sequestering the Standard Model Vacuum Energy," Phys. Rev. Lett. 112 no. 9, (2014) 091304, arXiv:1309.6562 [hep-th].

[64] A. De Felice and A. Naruko, "On metric transformations with a $U(1)$ gauge field," Phys. Rev. D 101 no. 8, (2020) 084044, arXiv:1911.10960 [gr-qc].

[65] R. Bufalo, J. Klusoň, and M. Oksanen, "Canonical formulation and path integral for local vacuum energy sequestering," Phys. Rev. D 94 no. 4, (2016) 044005, arXiv: 1606.01282 [hep-th].

[66] J. Klusoň, "Note About Canonical Formalism for Normalized Gravity And Vacuum Energy Sequestering Model," JHEP 01 (2015) 085, arXiv:1411.7501 [hep-th].

[67] A. Svesko and G. Zahariade, "On the Constraint Structure of Vacuum Energy Sequestering," JCAP 12 (2019) 033, arXiv:1812.11625 [hep-th].

[68] G. E. Volovik, "Varying Newton constant and black hole to white hole quantum tunneling," Universe $\mathbf{6}$ no. 9, (2020) 133, arXiv:2003.10331 [gr-qc].

[69] F. R. Klinkhamer and G. E. Volovik, " $\mathrm{f}(\mathrm{R})$ cosmology from q-theory," JETP Lett. 88 (2008) 289-294, arXiv:0807.3896 [gr-qc].

[70] S. M. Carroll and G. N. Remmen, "A Nonlocal Approach to the Cosmological Constant Problem," Phys. Rev. D 95 no. 12, (2017) 123504, arXiv:1703.09715 [hep-th].

[71] C. Deffayet, O. Pujolas, I. Sawicki, and A. Vikman, "Imperfect Dark Energy from Kinetic Gravity Braiding," JCAP 10 (2010) 026, arXiv: 1008.0048 [hep-th].

[72] T. Kobayashi, M. Yamaguchi, and J. Yokoyama, "G-inflation: Inflation driven by the Galileon field," Phys. Rev. Lett. 105 (2010) 231302, arXiv:1008.0603 [hep-th].

[73] C. Armendariz-Picon, V. F. Mukhanov, and P. J. Steinhardt, "Essentials of k essence," Phys. Rev. D 63 (2001) 103510, arXiv:astro-ph/0006373.

[74] T. Chiba, T. Okabe, and M. Yamaguchi, "Kinetically driven quintessence," Phys. Rev. D 62 (2000) 023511, arXiv: astro-ph/9912463.

[75] N. Kaloper and A. Padilla, "Vacuum Energy Sequestering: The Framework and Its Cosmological Consequences," Phys. Rev. D 90 no. 8, (2014) 084023, arXiv:1406.0711 [hep-th]. [Addendum: Phys.Rev.D 90, 109901 (2014)].

[76] N. Kaloper and A. Padilla, "Vacuum Energy Sequestering and Graviton Loops," Phys. Rev. Lett. 118 no. 6, (2017) 061303, arXiv:1606.04958 [hep-th].

[77] I. Ben-Dayan, R. Richter, F. Ruehle, and A. Westphal, "Vacuum energy sequestering and conformal symmetry," JCAP 05 (2016) 002, arXiv: 1507.04158 [hep-th] .

[78] E. A. Lim, I. Sawicki, and A. Vikman, "Dust of Dark Energy," JCAP 1005 (2010) 012, arXiv:1003.5751 [astro-ph.CO].

[79] T. Jacobson, "Thermodynamics of space-time: The Einstein equation of state," Phys. Rev. Lett. 75 (1995) 1260-1263, arXiv:gr-qc/9504004.

[80] T. Padmanabhan, "General Relativity from a Thermodynamic Perspective," Gen. Rel. Grav. 46 (2014) 1673, arXiv:1312.3253 [gr-qc].

[81] T. Padmanabhan, "Gravity and Quantum Theory: Domains of Conflict and Contact," arXiv:1909.02015 [gr-qc] .

[82] A. Alonso-Serrano and M. Liška, "Quantum phenomenological gravitational dynamics: A general view from thermodynamics of spacetime," JHEP 20 (2020) 196, arXiv:2009.03826 [gr-qc].

[83] S. Nagy, A. Padilla, and I. Zavala, "The Super-Stückelberg procedure and dS in pure supergravity," Proc. Roy. Soc. Lond. A $\mathbf{4 7 6}$ no. 2237, (2020) 20200035, arXiv:1910.14349 [hep-th].

[84] L. Baulieu, "Unimodular Gauge in Perturbative Gravity and Supergravity," Phys. Lett. B 808 (2020) 135591, arXiv:2004.05950 [hep-th].

[85] S. Bansal, S. Nagy, A. Padilla, and I. Zavala, "Unimodular vs Nilpotent Superfield Approach to Pure 
dS Supergravity," JHEP 01 (2021) 146, arXiv:2010.13758 [hep-th].

[86] M. Luscher, "The Secret Long Range Force in Quantum Field Theories With Instantons," Phys. Lett. B 78 (1978) 465-467.

[87] G. Dvali, "Three-form gauging of axion symmetries and gravity," arXiv:hep-th/0507215 [hep-th].
[88] G. Dvali, R. Jackiw, and S.-Y. Pi, "Topological mass generation in four dimensions," Phys. Rev. Lett. 96 (2006) 081602, arXiv:hep-th/0511175 [hep-th].

[89] G. Dvali, "A Vacuum accumulation solution to the strong CP problem," Phys. Rev. D74 (2006) 025019, arXiv:hep-th/0510053 [hep-th]. 